\title{
Adaptive Spatial Modulation over MmWave Channel with UPA
}

\author{
Yuhong Liu, Duoying Zhang* \\ College of Information Science and Technology, Jinan University, Guangzhou, China \\ Email address: \\ liuyuhong@stu2018.jnu.edu.cn (Yuhong Liu),johnson-zdy@qq.com (Duoying Zhang) \\ *Corresponding author
}

\section{To cite this article:}

Yuhong Liu, Duoying Zhang. Adaptive Spatial Modulation over MmWave Channel with UPA. International Journal of Information and Communication Sciences. Vol. 5, No. 3, 2020, pp. 33-40. doi: 10.11648/j.ijics.20200503.12

Received: September 2, 2020; Accepted: September 18, 2020; Published: September 25, 2020

\begin{abstract}
Spatial modulation (SM) utilizes the transmit antenna index and a quadrature amplitude modulation (QAM) symbol chosen from a constellation diagram to improve spectral efficiency (SE) comparing to conventional modulation scheme. However, in the conventional SM system, the modulation mode applied to the active antenna is fixed, which degrades the bits error rates (BER) and spectral efficiency (SE) performance. Moreover, a large number of researches on SM systems focus on the utilization of the uniform linear array (ULA) for transmission, which only considers the transmission on the horizontal domain while ignoring that on the vertical domain. Therefore, in this paper, we propose an adaptive spatial modulation (ASM) scheme with uniform planar array (UPA) over millimeter wave (mmWave) channels, which combines SM with adaptive modulation (AM) to enhance the performance of SE. To further improve the bits error rates (BER) performance, we develop an UPA-based ASM scheme with transmit antenna selection (TAS). We then analyse the BER and SE performance of both the two ASM scheme and obtain the closed-form expression for SE of the UPA-based ASM scheme with TAS algorithm. The simulations demonstrate that the proposed ASM schemes can achieve a considerable SE and a relatively low BER.
\end{abstract}

Keywords: ASM, UPA, mmWave, Multiple-input-multiple-output (MIMO)

\section{Introduction}

Nowadays, the spectrum at microwave frequency bands becomes scarcely, which limits data rates, whereas the spectrum in the millimeter wave (mmWave) realm ranging from $30 \mathrm{GHz}$ to $300 \mathrm{GHz}$ is abundant. MmWave communication is a promising way to alleviate the spectrum gridlock and offer gigabits-per-second data rate [1-8]. Due to the dramatic decrease of wavelength, a large number of antennas can be packed at transmitters in mmWave communication systems, which makes multiple-input-multiple-output (MIMO) powerful to compensate for the severe free-space pathloss of mmWave signals. However, with the number of radio frequency (RF) increasing, the high complexity and the cost of hardware and power consumption jeopardizes the attainable spectral efficiency (SE) gain provided by the mmWave-MIMO communication systems.

Spatial modulation (SM) is a promising modulation scheme to achieve high data rates with low complexity and power consumption due to the utilization of a single RF chain [9-15]. The conventional SM activates one antenna from the set of transmit antennas during each time slot and sends a symbol chosen from quadrature amplitude modulation (QAM) constellation diagram. Inter-antenna synchronization (IAS) and inter-channel interference (ICI) can be avoided with one activated antenna. The order of QAM alphabet needed in SM is lower than that in the conventional MIMO at the same SE because additional information bits are sent by spatial domain [16-18]. However, channel state information (CSI) may be not available to the transmitter in conventional SM system, which may degrade the performance of SE and bits error rates (BER). Adaptive modulation (AM) is a significant technique, which enables to alleviate adverse effects of deep fading of channel for the sake of the performance of symbol error rate (SER) or BER [19-21]. Adaptive spatial modulation (ASM) schemes combining AM with SM in. 


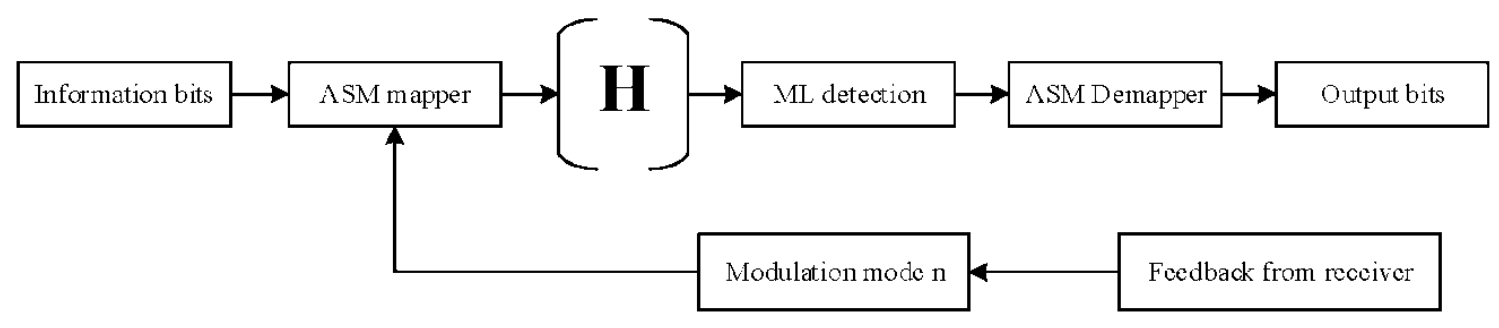

Figure 1. System Model.

MIMO communication systems are proposed in [22-25]. with the purpose of BER minimization at fixed data rates. The ASM scheme is designed to improve BER performance with an exhaustive search of the optimal transmission mode according to the CSI, which is of high complexity [22]. ASM schemes are dedicated to reduce the complexity with the exhaustive search strategy by shrinking the search space [23, 24]. Both the modulation order assigned to each antenna and the number of activated antennas are adaptive to channel conditions [25]. The ASM scheme adjusts the modulation mode with CSI to ensure that BER is under the given BER target [26]. ASM schemes aforementioned are based on uniform linear array (ULA), which only considers the horizontal characteristics of channel.

In this work, we propose ASM schemes in mmWave channel with uniform planar array (UPA) (UPA-based ASM), which can achieve transmission in both azimuth and elevation domains. The proposed UPA-based ASM scheme adjusts the modulation mode according to the CSI with the aim of SE improvement while maintaining the BER under the acceptable BER target. The proposed UPA-based ASM scheme exploits the first several information bits to select a column of antennas from UPA and additional information bits to choose the activated antenna from the selected column of transmitter antennas. The maximum achievable modulation mode for the activated antenna is chosen according to the feedback from the receiver. However, with the increase of the number of transmit antennas, the complexity of detection increases correspondingly, which may result in the degradation of BER performance. In order to reduce the complexity of detector and improve BER performance, we further develop an UPA-based ASM scheme with transmit antenna selection (TAS-UPA-based ASM scheme), in which a column of antennas is selected according to source bits while the activated antenna is chosen from that column of antennas via transmit antenna selection (TAS) algorithm. Simulation results illustrate the efficiency of the proposed ASM schemes in both SE and BER performances.

The remainder of the paper is arranged as follows. Section II mainly introduces the system model and the channel model. Section III illustrates two variants of UPA-based ASM schemes over mmWave-MIMO system in detail. Simulation results are produced in Section IV and the paper is concluded in Section V.

All notations in this paper are following. The transpose and the conjugate transpose are denoted by $(\cdot)^{\mathrm{T}}$ and $(\cdot)^{\mathrm{H}}$, respectively. The Frobenius norm of a vector or a matrix is denoted as $\|\cdot\|_{F}$. The real part of complex variable is denoted as $R e\{\cdot\}$. A complex Gaussia distribution of a random variable is denoted as $\mathcal{C N}\left(\mu, \sigma^{2}\right)$. The Kronecker product is denoted as $\otimes$.

\section{System Model}

\subsection{System Model}

We consider an mmWave-MIMO system with $\mathrm{N}_{\mathrm{t}}$ transmit antennas and $\mathrm{N}_{\mathrm{r}}$ receive antennas, which is displayed in Figure 1. The input information bits are splited to two parts. The first data bits determine the index of the chosen column of antennas. The other data bits are mapped to a constant-power variable-rate uncoded QAM symbol, which will be transmitted by the selected antenna. Different from the conventional SM scheme, the modulation mode is changing according to the feedback from receivers in order to send more data when the channel condition is good. Assuming that the selected transmit antenna locates at the $k$-th column and $l$-th row in the UPA configuration, $\mathrm{x} \in \mathbf{C}^{\mathrm{N} \times 1}$ denotes the transmit symbol vector, in which only one nonzero element $\mathrm{x}_{\mathrm{m}}$ drawn from the $M$-QAM constellation diagram is transmitted by the selected transmit antenna. The $(k, l)$-th antenna is corresponding to the $q$-th column of the channel matrix $\mathbf{H}$ where $q=k+\mathrm{N}_{\mathrm{th}}(l-1)$. ML detection is exploited at the receiver end. The $\mathrm{N}_{\mathrm{r}} \times 1$ received vector $\mathbf{y}$ is given by

$$
\mathbf{y}=\sqrt{\rho} \mathbf{H}_{q} \mathbf{x}_{m}+\mathbf{n}
$$

where $\rho$ is the average SNR of each receive antenna and $\mathbf{n}$ $\in \mathbb{C}^{\mathrm{N} \times 1}$ denotes additive white Gaussian noise (AWGN) with zero mean and variance $\sigma_{\mathrm{n}}^{2}$, that is, $\mathbf{n} \sim \mathcal{C N}\left(0, \sigma_{\mathrm{n}}^{2} \mathrm{I}\right)$

\subsection{Channel Model}

The mmWave signals are susceptible to shadowing and have high free-space loss [27]. The Saleh-Valenzuela model depicts the sparse scattering of the mmWave wireless propagation [28]. The channel matrix $\mathbf{H} \in \mathbf{C}^{\mathrm{N} \times \mathrm{Nt}}$ between the basestation (BS) and a user is defined as follows $[29,30]$.

$$
\mathbf{H}=\sqrt{\frac{N_{t} N_{r}}{N_{c l} N_{r a y}}} \sum_{i=1}^{N_{c l}} \sum_{j=1}^{N_{r a y}} \alpha_{i j} \mathbf{a}_{\mathbf{r}}\left(\phi_{i j}^{r}, \theta_{i j}^{r}\right) \mathbf{a}_{\mathbf{t}}^{\mathrm{H}}\left(\phi_{i j}^{t}, \theta_{i j}^{t}\right)
$$

where $N_{t}$ and $N_{r}$ denote the number of antennas at the transmitter and the receiver respectively, $\mathrm{N}_{\mathrm{cl}}$ and $\mathrm{N}_{\mathrm{ray}}$ denote 
the number of clusters and rays of each cluster respectively, $\alpha_{\mathrm{ij}}$ represents the complex gain of the $\mathrm{j}$-th ray in the $\mathrm{i}$-th cluster, $\mathbf{a}_{\mathbf{t}}\left(\phi_{\mathrm{ij}}^{\mathrm{t}}, \theta_{\mathrm{ij}}^{\mathrm{t}}\right)$ and $\mathbf{a}_{\mathbf{r}}\left(\phi_{\mathrm{ij}}^{\mathrm{r}}, \theta_{\mathrm{ij}}^{\mathrm{r}}\right)$ denote the array response on the transmitter and the user side respectively, $\phi_{\mathrm{ij}}^{\mathrm{t}}\left(\phi_{\mathrm{ij}}^{\mathrm{r}}\right)$ and $\theta_{\mathrm{ij}}^{\mathrm{t}}\left(\theta_{\mathrm{ij}}^{\mathrm{r}}\right)$ denote the azimuth angle of departure (the azimuth angle of arrival) and the elevation angle of departure (the elevation angle of arrival) respectively. Generally speaking, all of the clusters obey uniformly distribution, and rays in one cluster follow Laplace distribution in their own angle spread. $\alpha_{i j}$ is supposed to be independent identically distributed (i.i.d). and follows the distribution $\left(0, \sigma_{2 a, i}\right)$, where $\sigma_{2 a, i}$ is the average power of the $i$-th cluster.

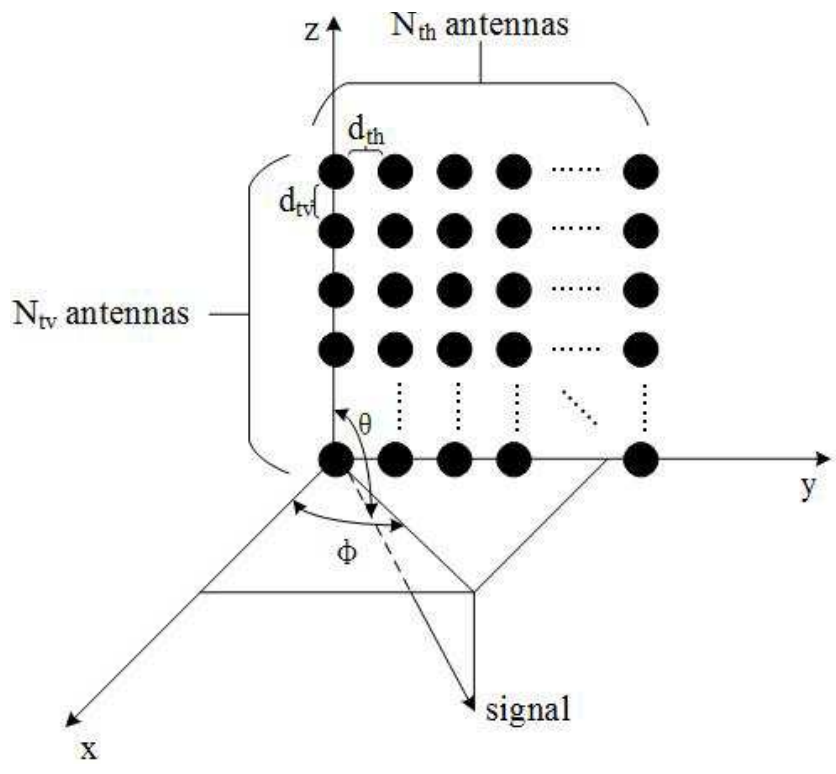

Figure 2. UPA.

In this paper, UPA is implemented at transmitter with Nth and $\mathrm{N}_{\mathrm{tv}}$ antenna elements on the horizontal and vertical domains, respectively, as shown in Figure 2. For simplification, the subscripts of departure angles on both azimuth and elevation are omitted and the steering vectors of UPA at the transmitter can be written as follows,

$$
\mathbf{a}_{\mathbf{t}}(\phi, \theta)=\frac{1}{\sqrt{N_{t}}} \mathbf{a}_{\mathbf{t h}}(\phi, \theta) \otimes \mathbf{a}_{\mathbf{t v}}(\phi, \theta)
$$

where $a_{t h}$ and $a_{t v}$ represent steering vectors on azimuth and elevation respectively

$$
\mathbf{a}_{\mathbf{t h}}(\phi, \theta)=\left[1, e^{j u d_{h} \sin \phi \cos \theta}, \ldots, e^{j u d_{h}\left(N_{t h}-1\right) \sin \phi \cos \theta}\right]^{T}
$$

and

$$
\mathbf{a}_{\mathbf{t v}}(\phi, \theta)=\left[1, e^{j u d_{v} \sin \phi \sin \theta}, \ldots, e^{j u d_{v}\left(N_{t v}-1\right) \sin \phi \sin \theta}\right]^{T}
$$

with $u=2 \pi / \lambda$. The carrier wavelength is denoted as $\lambda$ and $d_{h}$ $\left(d_{v}\right)$ represents interval spacing between adjacent antennas on horizontal (vertical) domain.

\subsection{Adaptive Threshold}

The BER of $M$-QAM with the received SNR, $\gamma$, can be approximated as [31],

$$
B E R_{n}(\gamma) \approx 0.2 e^{-\frac{3}{2\left(2^{n}-1\right)} \gamma}
$$

in which $M=2^{\mathrm{n}}$ is the modulation mode for the activated antenna.

The range of received SNR is separated into $\mathrm{N}+1$ regions. Given the target BER, $B E R_{t}$, the threshold, $\gamma_{\mathrm{n}}$, for the $(n+1)$-th region can be obtained by,

$$
\gamma_{n}=-\frac{2}{3} \ln \left(5 B E R_{t}\right)\left(2^{n}-1\right) \quad n=0,1, \ldots, N
$$

The modulation mode switches to $2^{n}$-QAM if $\gamma_{\mathrm{n}}<\gamma \leq \gamma_{\mathrm{n}+1}$.

\subsection{Maximum Likelihood (ML) Detection}

ML detection detects both the activated antenna index and symbol transmitted from that antenna jointly in SM transmission system [32]. ML detector of SM can be written as follows with $\mathrm{g}_{\mathrm{q}, \mathrm{m}}=\mathrm{H}_{\mathrm{q}} \mathrm{x}_{\mathrm{m}}$,

$$
\left[\hat{q}, \hat{x}_{m}\right]=\underset{q, m}{\arg \min } \sqrt{\rho}\left\|\mathbf{g}_{q, m}\right\|_{F}^{2}-2 \operatorname{Re}\left(\mathbf{y}^{H} \mathbf{g}_{q, m}\right)
$$

where $(\hat{q}, \hat{m})$ represent the combination of the estimated column and row index of the activated antenna and the symbol sent by the activated antenna, respectively.

\section{UPA-Based Scheme}

Most of SM schemes implement ULA antenna as antenna array configuration at the transmitter side without considering the vertical direction. It has been indicated that the antenna array configuration has an important impact on MIMO performance, which motivates us to explore the additional dimension [33]. Moreover, in the conventional SM, the information are divided into two parts, one part is mapped onto the conventional constellation points in signal domain, and the other part is used to determine the index of activated antenna in space domain. To overcome the deep fading, we propose the UPA-based ASM scheme to maximize the achievable modulation mode according to the channel quality while maintaining the acceptable BER level.

\subsection{UPA-based ASM Scheme}

\subsubsection{Bit Mapping in Space Domain}

Considering the additional dimension on vertical direction, the number of bits that can be transmitted via space domain can be expressed as,

$$
m_{S D}=\log _{2}\left(N_{t h}\right)+\log _{2}\left(N_{t v}\right)
$$

Referring to $[11,34]$, the bit mapping in space domain can be implemented as the following steps.

1) Select one of columns of UPA antenna according to imput bits with the length of $\log _{2}\left(N_{t h}\right)$

2) Activate one antenna from the selected column based on 
the input bits with the length of $\log _{2}\left(N_{t v}\right)$

\subsubsection{Bit Mapping in Signal Domain}

1) Select the maximum modulation mode $M$ according to the feedback of received SNR, based on (7)

2) Map the input bits into the selected modulation mode with the length of $\log _{2}(M)$.

Therefore, the total number of transmitted bits $m$ via the activated antenna can be written as,

$$
m_{\text {all }}=m_{S D}+\log _{2}(M)=\log _{2}\left(N_{t h}\right)+\log _{2}\left(N_{t v}\right)+\log _{2}(M)
$$

The corresponding SE can be improved due to the additional dimension on vertical direction, compared with ULA configuration.

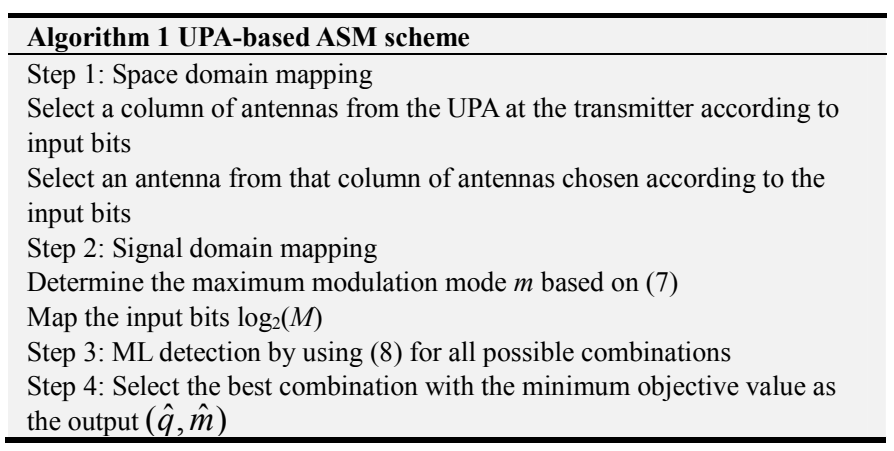

\subsection{TAS-UPA-based ASM Scheme}

In the previous subsection, the UPA-based ASM scheme selects the maximum achievable modulation mode to the channel quality under the acceptable BER level, in which the SE increases because of additional dimension on vertical direction. On the other hand, with the increase of the number of transmit antennas, it suffers from the high complexity of exhaustive search among all the transmit antenna candidates, and the high error probability that ML detection estimates the antenna index. As a result, we further develop a UPA-based ASM scheme that selects the transmit antenna via TAS algorithm.

Considering the channel quality, the bit mapping in space domain can be proposed as follows,

1) Select one of columns of UPA antenna according to input bits with the length of $\log _{2}\left(N_{t h}\right)$

2) Activate one antenna from the selected column via the TAS algorithm that can maximize the achievable sum rates, that is,

$$
\hat{\mathbf{h}}_{s e l}=\underset{s e l \in\left\{1, \ldots, N_{t v}\right\}}{\arg \max }\left\|\mathbf{h}_{\text {sel }}\right\|_{F}^{2}
$$

The bit mapping in signal domain follows the same steps of the UPA-based ASM scheme to select the maximum achievable modulation mode under the BER requirement. The details of the TAS-UPA-based ASM scheme can be found in Algorithm 2. input bits

Select an antenna from that column of antennas based (11)

Step 2: Signal domain mapping

Determine maximum modulation mode $\mathrm{m}$ based on (7)

Map the input bits $\log _{2}(M)$

Step 3: ML detection by using (8) for all possible combinations

Step 4: Select the best combination with the minimum objective value as the output $(\hat{q}, \hat{m})$

\section{Performance Analysis}

\subsection{SE Performance Analysis}

In both the two ASM schemes, an active antenna is utilized to transmit information. Therefore, the receiver SNR, $\gamma$, is a $\chi^{2}$-distributed random variable with $2 \times \mathrm{N}_{\mathrm{r}}$ degrees of freedom. The probability density function (PDF) of $\gamma$ is written in [26] as follows,

$$
f(\gamma)=\frac{\gamma^{N_{r}-1}}{\left(N_{r}-1\right) ! \rho^{N_{r}}} e^{-\frac{\gamma}{\rho}}
$$

Thus, the cumulative distribution function (CDF) of the received SNR, $\gamma$, can be written as follows,

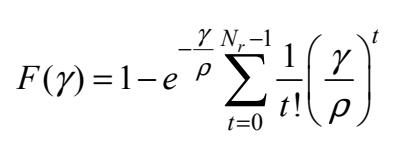

In the proposed two ASM schemes, the modulation order changes with the CSI. When the received SNR, $\gamma$, satisfies the requirement, $\gamma_{\mathrm{n}} \leq \gamma<\gamma_{\mathrm{n}+1}$, the modulation order applied to the active antenna for transmission is denoted as $2^{\mathrm{n}}$-QAM. The spectral efficiency is equivalent to the probability of the received SNR, $\gamma$, selecting the modulation order $2^{\mathrm{n}}$-QAM multiplies the data bits of each symbol under such a modulation mode. Therefore, the spectral efficiency obtained by the symbol domain, $\eta_{\mathrm{s}}$, is shown as follows,

$$
\eta_{s}=\sum_{n=1}^{N} n p_{n}
$$

\subsubsection{SE of UPA-based ASM Scheme}

In the UPA-based ASM scheme, the modulation order for transmission is determined by the maximal value $n$ that the received SNR can reach. Thus, the probability of selected $2^{n}$ as the modulation order can be derived as follows,

$$
p_{n}^{A S M}=\mathrm{P}\left\{\gamma_{\mathrm{n}} \leq \gamma<\gamma_{\mathrm{n}+1}\right\}=\mathrm{F}\left(\gamma_{\mathrm{n}+1}\right)-\mathrm{F}\left(\gamma_{\mathrm{n}}\right)
$$

Given that the probability is known, we can further obtain the spectral efficiency in the symbol domain for the UPA-based ASM scheme, $\eta_{s}^{A S M}$, which is written as follows,

$$
\eta_{s}^{A S M}=N-\sum_{n=2}^{N} F\left(\gamma_{n}\right)
$$

For the UPA-based ASM scheme, the column and row 
index of the antenna and amplitude and phase modulation symbol carry information bits. The overall spectral efficiency of the UPA-based ASM scheme, $S E^{\mathrm{ASM}}$, is derived as follows,

$$
\begin{aligned}
S E^{A S M} & =\log _{2} \mathrm{~N}_{\mathrm{th}}+\log _{2} \mathrm{~N}_{\mathrm{tv}}+\eta_{1} \\
& =\log _{2} \mathrm{~N}_{\mathrm{th}}+\log _{2} \mathrm{~N}_{\mathrm{tv}}+\mathrm{N}-\sum_{\mathrm{n}=2}^{\mathrm{N}} \mathrm{F}\left(\gamma_{\mathrm{n}}\right) .
\end{aligned}
$$

\subsubsection{SE of TAS-UPA-based ASM Scheme}

Different from the UPA-based ASM scheme, the antenna column index is determined by the information bits while the activated antenna is further selected by the TAS algorithm. Thus, the probability of selecting the $2^{n}$ as the modulation order can be derived as follows,

$$
p_{n}^{T A S-A S M}=\mathrm{P}\left\{\gamma_{\mathrm{n}} \leq \max _{\mathrm{i}=1, \cdots, \mathrm{N}_{\mathrm{tv}}} \gamma_{\mathrm{i}}<\gamma_{\mathrm{n}+1}\right\}=\left(\mathrm{F}\left(\gamma_{\mathrm{n}+1}\right)\right)^{\mathrm{N}_{\mathrm{tv}}}-\left(\mathrm{F}\left(\gamma_{\mathrm{n}}\right)\right)^{\mathrm{N}_{\mathrm{tv}}}
$$

With the probability known, we can further get the spectral efficiency in the symbol domain for TAS-UPA-based ASM scheme, $\eta_{s}^{T A S-A S M}$, which is given by,

$$
\eta_{s}^{T A S-A S M}=N-\sum_{n=2}^{N}\left(F\left(\gamma_{n}\right)\right)^{N_{t v}}
$$

Different from UPA-based ASM scheme, the TAS-UPA-based ASM scheme only uses the column index of the activated antenna and the signal constellation for transmission. Thus the SE of the TAS-UPA-based ASM scheme, $S E^{T A S-A S M}$, can be obtained by,

$$
S E^{T A S-A S M}=\log _{2} N_{t h}+\eta_{2}=\log _{2}\left(N_{t h}\right)+N-\sum_{n=2}^{N}\left(F\left(\gamma_{n}\right)\right)^{N_{t v}}
$$

\subsection{BER Performance Analysis}

The probability of perfect detection can be obtained by,

$$
P_{c}=(1-B E R a)(1-B E R s)
$$

where $\mathrm{BER}_{\mathrm{a}}$ and $\mathrm{BER}_{\mathrm{s}}$ denote the error rates of the antenna detection and the symbol detection, respectively at the assumption that either the antenna detection or the symbol detection is error. However, the BER performance of ASM scheme is decided by not only the estimated index of the transmitted antenna but also the transmitted symbol. Thus, the lower bound of the overall error detection rates, $B E R$, is given by,

$$
B E R \geq B E R_{a}+B E R_{s}-B E R_{a} B E R_{s}
$$

\subsubsection{Symbol Estimation BER}

The BER of symbol domain, $B E R_{s}$, is written as,

$$
B E R_{s}=\frac{1}{\eta_{s}} \sum_{n=1}^{N} n \overline{B E R_{n}}
$$

where $\overline{B E R_{n}}$ represents the average symbol BER with the
$2^{n}$-QAM selected for transmission, such as,

$$
\overline{B E R_{n}}= \begin{cases}\int_{0}^{\gamma_{2}} B E R_{1}(x) f(x) d x, & n=1 \\ \int_{\gamma_{n}}^{\gamma_{n+1}} B E R_{n}(x) f(x) d x, & n>1\end{cases}
$$

\subsubsection{Antenna Estimation BER}

The BER of spatial domain, $B E R_{\mathrm{a}}$, can be written as,

$$
B E R_{a}=\sum_{n=1}^{N} p_{n} B E R_{a \mid n}
$$

where $B E R_{a \mid n}$ denotes the error antenna detection conditioned of the estimated modulation order of $2^{n}$-QAM.

The lower bound of $B E R_{a \mid n}$ can be written as,

$$
B E R_{a \mid n} \leq\left\{\begin{array}{lc}
\sum_{q=1}^{N_{t}} \sum_{m=1}^{M} \sum_{\hat{q}=1}^{N_{t}} \frac{N_{1} P_{1}}{N_{t} M} & \text { for UPA-ASM scheme } \\
\sum_{l=1}^{N_{t v}} \sum_{m=1}^{M} \sum_{\hat{l}=1}^{N_{t v}} \frac{N_{2} P_{2}}{N_{t v} M} & \text { for } T A S-U P A-A S M \text { scheme }
\end{array}\right.
$$

where $N_{1}$ denotes the number of error bits between the transmit antenna index $q$ and the detected antenna index $\hat{q}$, $N_{2}$ represents the number of error bits between the column index of the selected antenna, 1, and the detected column index $\hat{l}, P_{1}$ and $P_{2}$ denote the probability of antenna index detection error and the column index error with perfect symbol detection, respectively.

\section{Simulation Results}

In this simulation, we compare the performance of the proposed two schemes, that is, the UPA-based ASM scheme and the TAS-UPA-based ASM scheme, with the ULA-based ASM scheme [26]. It is assumed that the delay and error of the feedback channel are zero. The initial parameters are set as follows, $B E R_{t}=10^{-3}$ and $\mathrm{N}=6$ which indicates that the modulation order can be adaptive among 2-QAM, 4-QAM, 8-QAM, 16-QAM, 32-QAM, 64-QAM.

\subsection{SE Performance}

Figure 3 presents the SE performance versus SNR. The proposed ASM schemes achieve higher SE than that of the ULA-based ASM scheme due to the additional elevation direction for transmission. The gap of SE between the UPA-based ASM scheme and the ULA-based ASM scheme is obvious in the low SNR region because the number of transmit antennas dominates the SE performance. With the application of TAS algorithm, a higher modulation mode $M$ can be achieved by the TAS-UPA-based ASM scheme compared to the ULA-based ASM scheme, which improves the SE performance. In the middle regime of SNR, the SE of the UPA-based ASM scheme is approaching to that of the ULA-based ASM scheme. It is because that the modulation mode $M$ increases slowly in the proposed UPA-based ASM scheme due to the degradation of BER performance brought 
by the increase of transmit antennas. Moreover, the SE performance is mainly determined by the signal domain, in which the highest SE is obtained by the TAS-UPA-based ASM scheme. In the high SNR region, when the SNR is larger enough to obtain the maximum modulation mode, that is, 64-QAM, the number of transmit antennas has main effect on SE. Therefore, the gap between the UPA-based ASM scheme and the rest of ASM schemes becomes larger.

\subsection{BER Performance}

Figure 4 demonstrates the BER performance versus SNR. The BER performance of the UPA-based ASM scheme is worse than that of the ULA-based ASM scheme, because the error probability of transmit antenna index detection increases with the number of transmit antennas. Moreover, an improvement of BER performance is obtained by the
TAS-UPA-based ASM scheme, compared to the rest of schemes, in which the TAS algorithm selects the channel that can maximize the achievable sum rates.

\subsection{Modulation Order}

Figure 5 shows the modulation mode $M$ versus SNR with different numbers of transmit antennas. As we expect, the modulation order increases with the SNR. The previous results indicate that the increase of transmit antennas degrades the BER performance, which leads to a slow growth of modulation order in the proposed UPA-based ASM scheme, compared to the ULA-based ASM scheme. In the TAS-UPA-based ASM scheme, the TAS algorithm select the best channel conditions and the highest modulation mode that can be obtained.

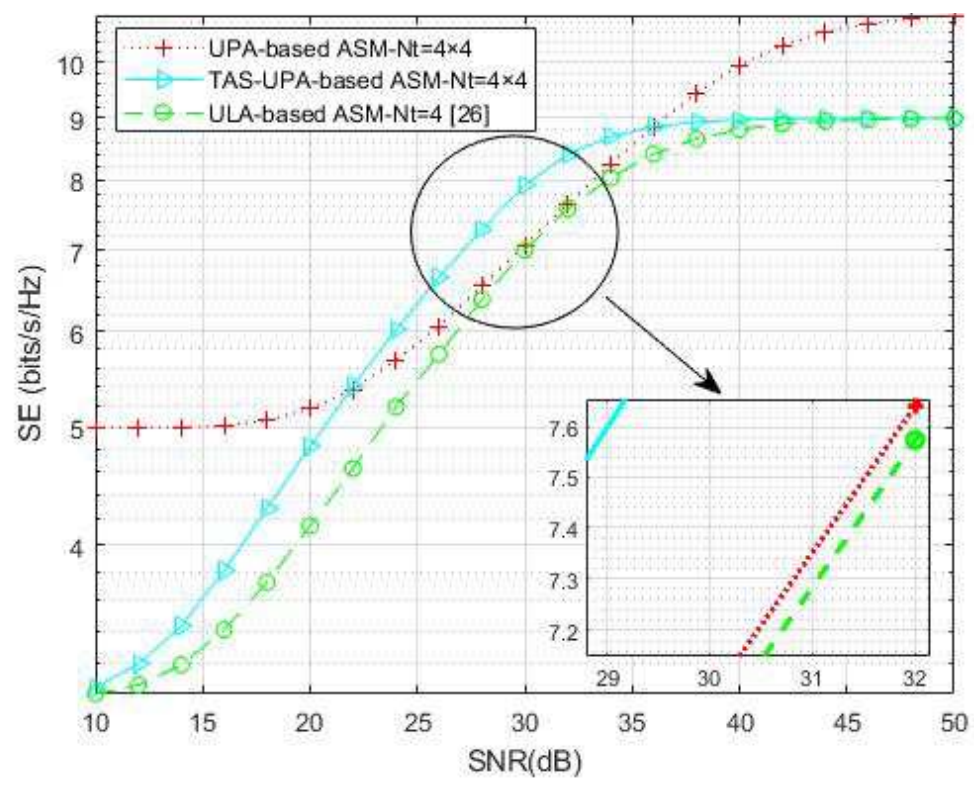

Figure 3. SE performance versus $S N R\left(N_{r}=2\right)$.

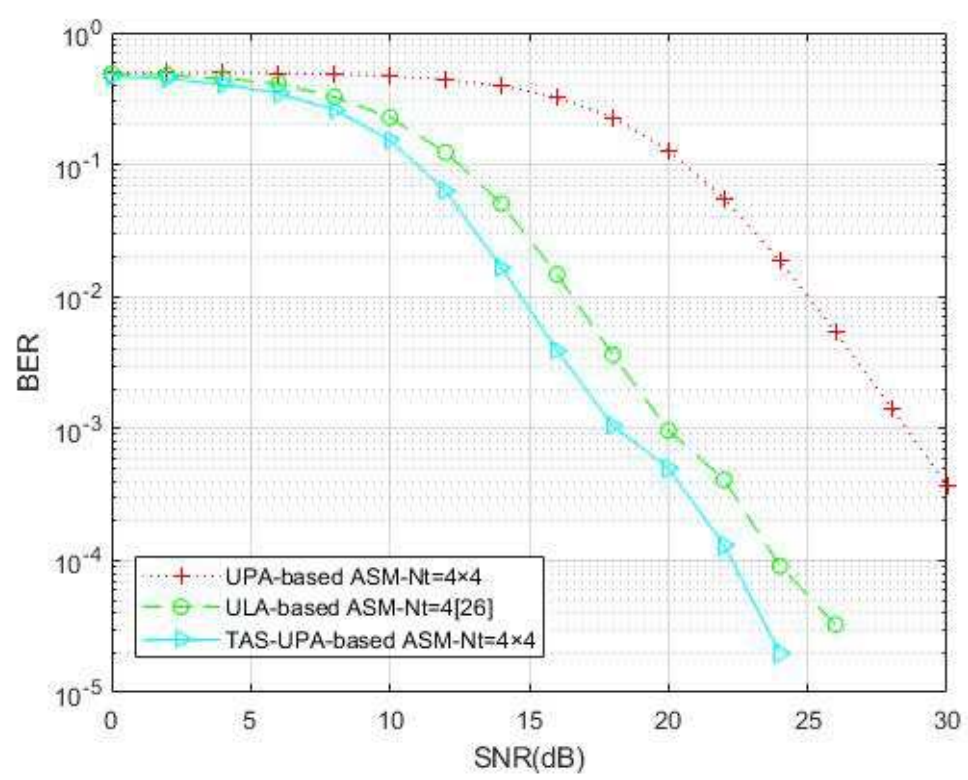

Figure 4. BER performance versus $S N R\left(N_{r}=2\right)$. 


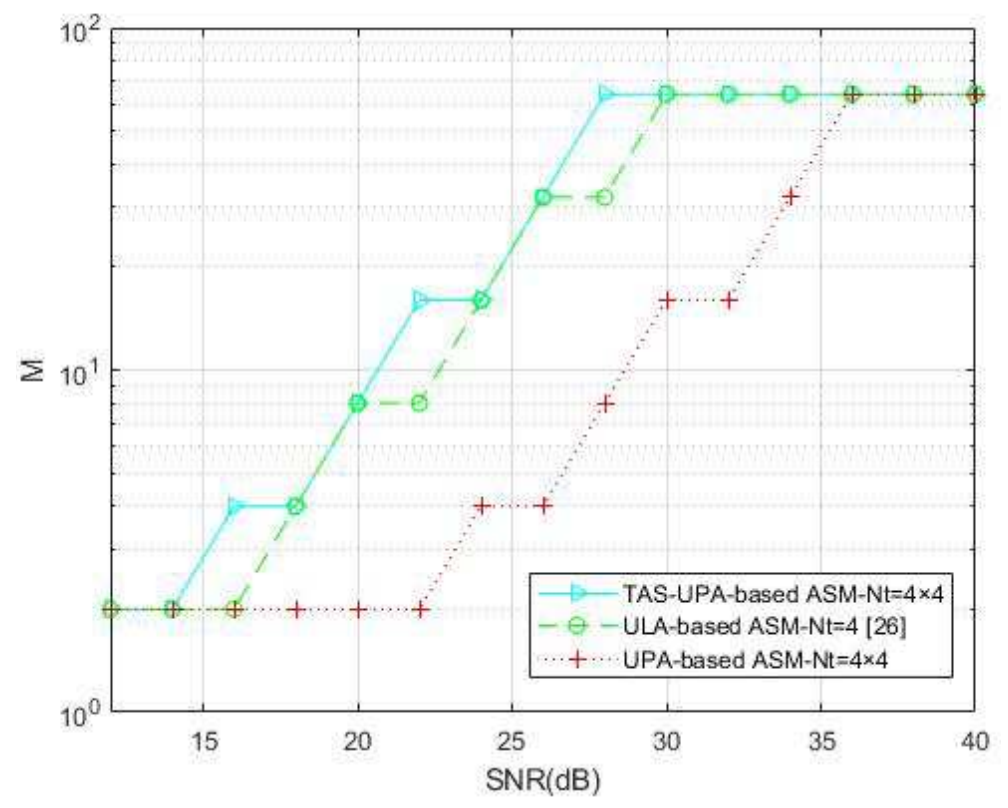

Figure 5. Modulation order M versus $S N R\left(N_{r}=2\right)$.

\section{Conclusion}

In this work, we propose the UPA-based ASM scheme and the TAS-UPA-based ASM scheme in mmWave-MIMO communication system. The proposed UPA-based ASM scheme utilizes both the horizontal and vertical domains for transmission to bring the enhancement on SE, in which the activated antenna to convey information is decided by random data bits In order to further improve BER performance, TAS algorithm is introduced to choose the activated antenna from the selected a column of antennas in the TAS-UPA-based ASM scheme. Due to the elevation direction for additional transmission, extra information bits can be conveyed in the proposed ASM schemes compared to the ULA-based ASM scheme.

\section{Acknowledgements}

The work of Yuhong Liu is supported by the National Nature Science Foundation of China (No. 61401178).

\section{References}

[1] L. Wei, R. Q. Hu, Y. Qian, and G. Wu, "Key elements to enable mil- limeter wave communications for $5 \mathrm{G}$ wireless systems," IEEE Wireless Communications, vol. 21, no. 6, pp. 136-143, 2014.

[2] F. Boccardi, R. W. Heath Jr, A. Lozano, T. L. Marzetta, and P. Popovski, "Five disruptive technology directions for 5G," arXiv preprint arXiv: 1312.0229, 2013.

[3] T. Bai, A. Alkhateeb, and R. W. Heath, "Coverage and capacity of millimeter-wave cellular networks," IEEE Communications Magazine, vol. 52, no. 9, pp. 70-77, 2014.

[4] A. Alkhateeb, J. Mo, N. Gonzalez-Prelcic, and R. W. Heath,
"MIMO precoding and combining solutions for millimeter-wave systems," IEEE Communications Magazine, vol. 52, no. 12, pp. 122-131, 2014.

[5] T. S. Rappaport, R. W. Heath Jr, R. C. Daniels, and J. N. Murdock, Millimeter wave wireless communications. Pearson Education, 2015.

[6] T. S. Rappaport, Y. Xing, G. R. MacCartney, A. F. Molisch, E. Mellios, and J. Zhang, "Overview of millimeter wave communications for fifth-generation (5G) wireless networks - with a focus on propagation models," IEEE Transactions on Antennas and Propagation, vol. 65, no. 12, pp. 6213-6230, 2017.

[7] I. A. Hemadeh, K. Satyanarayana, M. El-Hajjar, and L. Hanzo, "Millimeter-wave communications: Physical channel models, design considerations, antenna constructions, and link-budget," IEEE Communications Surveys \& Tutorials, vol. 20, no. 2, pp. 870-913, 2017.

[8] R. W. Heath, N. Gonzalez-Prelcic, S. Rangan, W. Roh, and A. M. Sayeed, "An overview of signal processing techniques for millimeter wave MIMO systems," IEEE journal of selected topics in signal processing, vol. 10, no. 3, pp. 436-453, 2016.

[9] Y. A. Chau and S. H. Yu, "Space modulation on wireless fading channels," in IEEE 54th Vehicular Technology Conference. VTC Fall 2001. Proceedings (Cat. No. 01CH37211), vol. 3. IEEE, 2001, pp. 1668-1671.

[10] H. Haas, E. Costa, and E. Schulz, "Increasing spectral efficiency by data multiplexing using antenna arrays," in The 13th IEEE International Symposium on Personal, Indoor and Mobile Radio Communications, vol. 2. IEEE, 2002, pp. 610613.

[11] R. Y. Mesleh, H. Haas, S. Sinanovic, C. W. Ahn, and S. Yun, "Spatial modulation," IEEE Transactions on vehicular technology, vol. 57, no. 4, pp. 2228-2241, 2008.

[12] A. Stavridis, S. Sinanovic, M. Di Renzo, and H. Haas, "Energy evaluation of spatial modulation at a multi-antenna base station," in 2013 IEEE 78th Vehicular Technology Conference (VTC Fall). IEEE, 2013, pp. 1-5. 
[13] M. Di Renzo, H. Haas, A. Ghrayeb, S. Sugiura, and L. Hanzo, "Spatial modulation for generalized MIMO: Challenges, opportunities, and implementation," Proceedings of the IEEE, vol. 102, no. 1, pp. 56-103, 2013.

[14] M. Wen, B. Zheng, K. J. Kim, M. Di Renzo, T. A. Tsiftsis, K. C. Chen, and N. Al-Dhahir, "A survey on spatial modulation in emerging wireless systems: Research progresses and applications," IEEE Journal on Selected Areas in Communications, vol. 37, no. 9, pp. 1949-1972, 2019.

[15] P. Yang, Y. Xiao, Y. L. Guan, K. Hari, A. Chockalingam, S. Sugiura, H. Haas, M. Di Renzo, C. Masouros, Z. Liu et al., "Single-carrier SM-MIMO: A promising design for broadband large-scale antenna systems," IEEE Communications Surveys \& Tutorials, vol. 18, no. 3, pp. 1687-1716, 2016.

[16] T. L. Narasimhan, P. Raviteja, and A. Chockalingam, "Large-scale multiuser SM-MIMO versus massive MIMO," in 2014 Information Theory and Applications Workshop (ITA). IEEE, 2014, pp. 1-9.

[17] N. Serafimovski, S. Sinanovic', M. Di Renzo, and H. Haas, "Multiple access spatial modulation," EURASIP Journal on Wireless Communications and Networking, vol. 2012, no. 1, p. 299, 2012.

[18] T. Datta, H. S. Eshwaraiah, and A. Chockalingam, "Generalized space and frequency index modulation," IEEE Transactions on Vehicular Technology, vol. 65, no. 7, pp. 4911-4924, 2015.

[19] C. Guo, "Research on adaptive modulation of MIMO MPSK systems," in 2010 Second International Conference on Networks Security, Wireless Communications and Trusted Computing, vol. 1. IEEE, 2010, pp. 390-393.

[20] J. F. Paris and A. J. Goldsmith, "Adaptive modulation for MIMO multiplexing under average BER constraints and imperfect CSI," in 2006 IEEE International Conference on Communications, vol. 3. IEEE, 2006, pp. 1318-1325.

[21] Z. Zhou, B. Vucetic, M. Dohler, and Y. Li, "MIMO systems with adaptive modulation," IEEE transactions on Vehicular Technology, vol. 54, no. 5, pp. 1828-1842, 2005.

[22] P. Yang, Y. Xiao, Y. Yu, and S. Li, "Adaptive spatial modulation for wireless MIMO transmission systems," IEEE Communications Letters, vol. 15, no. 6, pp. 602-604, 2011.

[23] P. Yang, Y. Xiao, Y. Yu, L. Li, Q. Tang, and S. Li, “Simplified adaptive spatial modulation for limited-feedback MIMO systems," IEEE Transactions on Vehicular Technology, vol. 62, no. 6, pp. 2656-2666, 2013.

[24] T. V. H. Nguyen, S. Sugiura, and K. Lee, "Low-complexity sphere search-based adaptive spatial modulation," IEEE Transactions on Vehicular Technology, vol. 67, no. 8, pp. 7836-7840, 2018.

[25] P. Yang, Y. Xiao, L. Li, Q. Tang, Y. Yu, and S. Li, "Link adaptation for spatial modulation with limited feedback," IEEE Transactions on Vehicular Technology, vol. 61, no. 8, pp. 3808-3813, 2012.

[26] Z. Bouida, A. Ghrayeb, and K. A. Qaraqe, "Adaptive spatial modulation for spectrally-efficient MIMO systems," in 2014 IEEE Wireless Communications and Networking Conference (WCNC). IEEE, 2014, pp. 583-587.

[27] S. Rangan, T. S. Rappaport, and E. Erkip, "Millimeter wave cellular wireless networks: Potentials and challenges," arXiv preprint arXiv: 1401.2560, 2014.

[28] A. A. Saleh and R. Valenzuela, "A statistical model for indoor multipath propagation," IEEE Journal on selected areas in communications, vol. 5, no. 2, pp. 128-137, 1987.

[29] O. El Ayach, S. Rajagopal, S. Abu-Surra, Z. Pi, and R. W. Heath, "Spatially sparse precoding in millimeter wave MIMO systems," IEEE transactions on wireless communications, vol. 13, no. 3, pp. 1499-1513, 2014.

[30] M. R. Akdeniz, Y. Liu, M. K. Samimi, S. Sun, S. Rangan, T. S. Rappaport, and E. Erkip, "Millimeter wave channel modeling and cellular capacity evaluation," IEEE journal on selected areas in communications, vol. 32, no. 6, pp. 1164-1179, 2014.

[31] M. S. Alouini and A. J. Goldsmith, "Adaptive modulation over nakagami fading channels," Wireless Personal Communications, vol. 13, no. 1-2, pp. 119-143, 2000.

[32] J. Jeganathan, A. Ghrayeb, and L. Szczecinski, "spatial modulation: Optimal detection and performance analysis." IEEE Communications Letters, vol. 12, no. 8, pp. 545-547, 2008.

[33] W. Tan, S. D. Assimonis, M. Matthaiou, Y. Han, X. Li, and S. Jin, "Analysis of different planar antenna arrays for mmWave massive MIMO systems," in 2017 IEEE 85th Vehicular Technology Conference (VTC Spring). IEEE, 2017, pp. 1-5.

[34] P. Yang, M. Di Renzo, Y. Xiao, S. Li, and L. Hanzo, "Design guidelines for spatial modulation," IEEE Communications Surveys \& Tutorials, vol. 17, no. 1, pp. 6-26, 2014. 